\title{
On The Residual Effective Potential within Global One-Dimensional Quantum Gravity
}

\author{
Lukasz Andrzej Glinka*
}

Member of the American Association of International Researchers. New York, USA

\begin{abstract}
The global one-dimensional quantum gravity is the model of quantum gravity which arises from the global onedimensionality conjecture within quantum general relativity, first considered by the author in 2010 and then in 2012. In this model the global dimension is a determinant of a metric of three-dimensional space embedded into an enveloping Lorentizan four-dimensional space-time. In 2012, it has already been presented by the author that this model can be extended to any Lorentzian $D+1$-dimensional space-time, where $D$ is a dimension of space, and resulting in the global one-dimensional model of a higher dimensional quantum gravity.

The purely quantum-mechanical part of this model is a minimal effective model within the quantum Geometrodynamics, introduced by J.A. Wheeler and B.S. DeWitt in the 1960s, but the effective potential is manifestly different from the one considered by Wheeler \& DeWitt. Moreover, in our model the wave functionals solving the quantum gravity are one-variable smooth functions and, therefore, the troublesome mathematical technique of the Feynman functional integration present in the Hawking formulation of quantum gravity, is absent is this model, what makes it a mathematically consistent theory of quantum gravitation.

In this paper, we discuss in some detail a certain part of the global one-dimensional model already proposed in 2010, and then developed in 2012. The generalized functional expansion of the effective potential and the residual approximation, which describe the embedded spaces which are maximally symmetric three-dimensional Einstein's manifolds, whose lead to the Newton-Coulomb type potential in the quantum gravity model, are considered. Furthermore, scenarios related to few selected specific forms of the effective potential are suggested as physically interesting and discussed.
\end{abstract}

Keywords: Global one-dimensionality conjecture; Quantum gravity model; Quantum geometrodynamics; Effective potential; Residual approximation

\section{Introduction}

It was recently proposed by the author, $[1,2]$, to take into account the global one-dimensionality conjecture within quantum general relativity, where the global dimension is determinant of metric of a three-dimensional space embedded into four-dimensional space-time. Actually, this conjecture leads to the non-trivial model of quantum gravity which differs from the standardly considered approaches [312].

The quantum-mechanical part of the global one-dimensional quantum gravity can be considered separately as a nice mathematical theory having possibly interesting physical ramifications. In and of itself this fragment of the model is globally one-dimensional quantum mechanics describing $3+1$-decomposed solutions of the Einstein field equations of general relativity. In this theory quantum gravity is given by the one-dimensional Schrödinger equation, where the single dimension is the global dimension. The global quantum mechanics can be interpreted in terms of radial-type Schrödinger wave equation and, for this reason; it straightforwardly leads to the strict physical relation with atomic and nuclear physics.

In this paper few selected elements of the globally one-dimensional quantum mechanics are discussed in some detail. The generalized functional expansion of the effective potential and the residual approximation of the expansion, which corresponds to the embedding being the maximally symmetric three-dimensional Einstein manifolds, whose the physical meaning is reconstruction the Newton-Coulomb type potential within the model of quantum gravity, are considered.
Few possible mathematical scenarios with respect to the form of the effective potential are suggested as possibly interesting from the theoretical physics point of view.

The content of this paper is as follows. In the Section 7 the global one-dimensional model of quantum gravity is briefly discussed, and its quantum mechanical part is presented in some detail. We start, in the Subsection 7.1, from the condensed presentation of the standard way resulting in the Wheeler-DeWitt equation of quantum Geometrodynamics which is the standardly considered model of quantum gravity and is the core fundament of all presently considered approaches. Then, in the Subsection 7.2, the idea of the global dimension, the resulting quantum mechanics, and the idea of the generalized functional expansion of the effective potential are presented. Next, in the Subsection 7.3, the idea of the invariant global dimension is digressed only, with no continuation in the further part of the paper. In the Section 8 , the residual approximation of the effective potential, the role of maximally symmetric three-dimensional Einstein manifolds within the model of quantum gravity, and few conclusions

*Corresponding author: Lukasz Andrzej Glinka, Member of the American Association of International Researchers. New York, USA; Tel: +48-660-019-237; E-mail: laglinka@gmail.com

Received February 05, 2014; Accepted February 25, 2014; Published March 05, 2014

Citation: Glinka LA (2014) On The Residual Effective Potential within Global One-Dimensional Quantum Gravity. J Astrophys Aerospace Technol 2: 103. doi:10.4172/2329-6542.1000103

Copyright: ( $) 2014$ Glinka LA. This is an open-access article distributed under the terms of the Creative Commons Attribution License, which permits unrestricted use, distribution, and reproduction in any medium, provided the original author and source are credited. 
having possibly interesting physical significance are discussed. The Section 7 is devoted to derivation of the "geometric" wave functionals of the global quantum mechanics in terms of the special functions, with respect to the selected situations given by the three types of boundary conditions discussed one by one in the Subsections 7.1, 7.2, and 7.3. Finally, in the Section 8 we summarize briefly the paper.

\section{Global One-Dimensional Quantum Gravity}

\section{Standard quantum geometrodynamics}

In general relativity $[13,14]$ a Lorentzian (pseudo-Riemannian) [15] manifold $(\mathrm{M}, \mathrm{g})$ with a metric and a distance $d s^{2}=g_{\mu \nu} d x^{\mu} d x^{\nu}$ , where $x^{\mu}, \mu=0,1,2,3$ is a coordinate system, characterized by the Christoffel symbols $\Gamma_{\mu \nu}^{p}$ and curvatures: Riemann-Christoffel $R_{\mu \alpha v^{\prime}}^{\lambda}$ Ricci Ricci $R_{\mu v^{\prime}}$ scalar $\mathrm{R}$

$$
\begin{aligned}
& \Gamma_{\mu v}^{p}=\frac{1}{2} g^{\sigma}(g \mu \sigma, v+g \sigma v, \mu-g \mu v, \sigma), \\
& R_{\mu \alpha v}^{\lambda}=\Gamma_{\mu v, \alpha}^{\lambda}-\Gamma_{\mu \alpha, v}^{\lambda}+\Gamma_{\sigma \alpha}^{\lambda} \Gamma_{\mu \nu}^{\sigma}-\Gamma_{\sigma v}^{\lambda} \Gamma_{\mu \alpha}^{\sigma}, \\
& R_{\mu v}=R_{\mu \lambda v}^{\lambda}, \\
& R=g^{\kappa \lambda} R_{\kappa \lambda},
\end{aligned}
$$

is a space time being a solution of the Einstein field equations

$$
G_{\mu v}+\Lambda g_{\mu v}=\kappa T_{\mu v},
$$

Where $\kappa$ is the Einstein constant, $G_{\mu v} \equiv R_{\mu v}-\frac{1}{2} R g_{\mu v}$ is the Einstein tensor, $\Lambda$ is cosmological constant, and $T_{\mu v}$ is stress-energy tensor of Matter fieldswhich arises through the variationprinciple [16] applied to the EinsteinHilbert action modified through the York-GibbonsHawking boundary term $[17,18]$

$$
S[g]=\int_{M} d \mu g\left\{-\frac{R}{2 \kappa}+\frac{\Lambda}{\kappa}+\mathscr{L}\right\}-\frac{1}{\kappa} \int_{\partial M} d \mu_{h} K,
$$

Where $\kappa$ is the Gauss scalar curvature of a spacelike boundary $\mathscr{L}_{1}$ is the Lagrangian of Matter fields, and $d \mu_{g}=d^{4} x \sqrt{-g}, d \mu_{h}=d^{3} x \sqrt{h}$ are the invariant measures of four-dimensional space-time and embedded three-dimensionalspace, respectively.

Application of the Nash embedding theorem [19-22] allows justifying the 3+1 decomposition of spacetime metric [23-25]

$$
g_{\mu v}=\left[\begin{array}{cc}
-N^{2}+N^{i} N_{i} & N_{j} \\
N_{i} & h_{i j}
\end{array}\right], \quad h_{i k} h^{k j}=\delta_{i}^{j},
$$

Where $N, N_{i}, h_{i j}$ are respectively called the lapse function, the shift vector, and embedded space metric, $N^{i}=h^{i j} N_{j}$ is the intrinsic covariant shift vector. The $3+1$ decomposition transforms the action functional (3) into the Hamiltonian form

$$
S[g]=\int d t \int_{\partial M} d^{3} x\left\{\pi_{\phi} \dot{\phi}+\pi \dot{N}+\pi^{i} \dot{N}_{i}+\pi^{i j} \dot{h}_{i j}-N H-N_{i} H^{i}\right\},
$$

Where $\phi$ symbolizes Matter fields, and nontrivial $\pi$ 's, $\mathrm{H}, \mathrm{H}^{\mathrm{i}}$ are [26]

$$
\begin{aligned}
& \pi^{i j}=-\frac{\sqrt{h}}{2 \kappa}\left(K^{i j}-K h^{i j}\right), \\
& H=\frac{\sqrt{h}}{2 \kappa}\left\{^{(3)} R+K^{2}-K_{i j} K^{i j}-2 \Lambda-2 \kappa e\right\}, \\
& H^{i}=2 \pi_{; j}^{i j},
\end{aligned}
$$

Where ${ }^{(3)} \mathrm{R}$ is the Ricci scalar of an embedding, $e=n^{\mu} n^{v} T_{\mu v}$

is the Matter fields energy related to the normal vector field $n^{\mu}=\left[1 / N,-N^{i} / N\right]$. Extrinsic curvature $K_{i j}$, where $\operatorname{Tr} K_{i j} \equiv K$, is constrained by the equality

$$
\dot{h}_{i j}=2\left(N K_{i j}+N_{(i \mid j)}\right) \text {, }
$$

Where is symmetrized intrinsic covariant derivative of the shift. In accordance with DeWitt's foundational considerations [27], $\mathrm{H}^{\mathrm{i}}$ are the generators of the spatial diffeomorphisms $\tilde{x}^{i}=x^{i}+\delta x^{i}$

$$
\begin{aligned}
& i\left[h_{i j}, \int_{\partial M} H_{a} \boldsymbol{\xi}^{a} d^{3} x\right]=-h_{i j, k} \boldsymbol{\xi}^{k}{ }_{, i}-h_{k j} \boldsymbol{\xi}^{k}{ }_{, i}-h_{i k} \boldsymbol{\xi}^{k}, \\
& i\left[\pi^{i j}, \int_{\partial M} H_{a} \boldsymbol{\xi}^{a} d^{3} x\right]=-\left(\pi^{i j} \boldsymbol{\xi}^{k}\right)_{, k}+\pi^{k j} \boldsymbol{\xi}_{, k}^{i}+\pi^{i k} \boldsymbol{\xi}_{, k}^{j},
\end{aligned}
$$

Where $H_{i}=h_{i j} H^{j}$. Time-preservation [28] of the primary constraints $\pi \approx 0$ and $\pi^{i} \approx 0$ leads to secondary ones-scalar and vector constraints

$$
\begin{aligned}
& H \approx 0, \\
& H^{i} \approx 0,
\end{aligned}
$$

Which create nontrivial first-class type constraints algebra [27]

$$
\begin{aligned}
& i\left[\int_{\partial M} H \delta x_{1} d^{3} x, \int_{\partial M} H \delta x_{2} d^{3} x\right]=\int_{\partial M} H^{a}\left(\delta x_{1, a} \delta x_{2}-\delta x_{1} \delta x_{2, a}\right) d^{3} x, \\
& i\left[H_{i}(x), H_{j}(y)\right]=\int_{\partial M} H_{a} c_{i j}^{a} d^{3} z, \\
& i\left[H(x), H_{i}(y)\right]=H \delta_{, i}^{(3)}(x, y),
\end{aligned}
$$

Where

$$
c_{i j}^{a}=c_{i j}^{a}[x, y, z]=\delta_{i}^{a} \delta_{j}^{b} \delta_{, b}^{(3)}(x, z) \delta^{(3)}(y, z)-(i \leftrightarrow j, x \leftrightarrow y),
$$

are structure constants of the diffeomorphism group and all Lie's bracketsof $\pi$ 's and H'svanish. Scalar constraint determines dynamics, vector one merely reflects diffeoinvariance. Making use of the conjugate momenta, first formula in (12), and the scalar constraint transforms into the Hamilton-Jacobi type equation

$$
H=G_{i j k l} \pi^{i j} \pi^{k l}+\sqrt{h}\left({ }^{(3)} R-2 \Lambda-2 \kappa e\right) \approx 0,
$$

Where

$$
G_{i j k l} \equiv \frac{1}{2 \sqrt{h}}\left(h_{i k} h_{j l}+h_{i l} h_{j k}-h_{i j} h_{k l}\right),
$$

is the DeWitt metric on the Wheeler super space, a factor space of all $c^{\infty}$ Riemannia metrics on $\partial M$, and a group of all $c^{\infty}$ diffeomorphisms of $\partial M$ that preserve orientation [29-31]. The Dirac-Faddeev primary canonical quantization method $[28,32]$ in the present case has the form

$$
\begin{aligned}
& i\left[\pi^{i j}(x), h_{k l}(y)\right]=\frac{1}{2}\left(\delta_{k}^{i} \delta_{l}^{j}+\delta_{l}^{i} \delta_{k}^{j}\right) \delta^{(3)}(x, y), \\
& i\left[\pi^{i}(x), N_{j}(y)\right]=\delta_{j}^{i} \delta^{(3)}(x, y), \\
& i[\pi(x), N(y)]=\delta^{(3)}(x, y) .
\end{aligned}
$$

The solutions or rather representations of the momenta operators satisfying these canonical commutation relations is the question of choice. In quantum Geometrodynamics, the Wheeler metric representation is usually taken into account. In such a representation the momenta operators are analogous to the momentum operator in quantum mechanics 


$$
\begin{aligned}
& \pi=-i \frac{\delta}{\delta N}, \\
& \pi^{i}=-i \frac{\delta}{\delta N_{i}}, \\
& \pi^{i j}=-i \frac{\delta}{\delta h_{i j}},
\end{aligned}
$$

and applied to the Hamiltonian constraint (12) lead to the WheelerDeWitt equation $[27,33]$

$$
\left\{2 \kappa G_{i j k l} \frac{\delta^{2}}{\delta h_{i j} \delta h_{k l}}+\frac{\sqrt{h}}{2 \kappa}\left({ }^{(3)} R-2 \Lambda-2 \kappa e\right)\right\} \Psi\left[h_{i j}, \phi\right]=0,
$$

and other first class constraints

$$
\begin{aligned}
& \pi \Psi\left[h_{i j}, \phi\right]=0, \\
& \pi^{i} \Psi\left[h_{i j}, \phi\right]=0, \\
& H^{i} \Psi\left[h_{i j}, \phi\right]=0,
\end{aligned}
$$

Merely reflect diffeoinvariance, and are not important for this model. Since about 60 years classical and quantum aspects of the Wheeler-DeWitt Geometrodynamics have studied widely (Cf. e.g. the books and collective volumes) in [3-12], and individual papers, [34-48].

\section{The global dimension}

The global one-dimensionality conjecture within quantum general relativity $[1,2]$ assumes the effective physical role of the determinant of a three-dimensional space embedded into a four-dimensional spacetime

$$
h=\operatorname{det} h_{i j}=\frac{1}{3} \varepsilon^{i j k} \varepsilon^{a b c} h_{i a} h_{j b} h_{k c},
$$

Where $\varepsilon^{i j k}$ is the Levi-Civita density, and takes into account the following situation as physically interesting

$$
\begin{aligned}
& \phi(x) \rightarrow \phi[h], \\
& e(\phi) \rightarrow e[h], \\
& \Psi\left[h_{i j}, \phi\right] \rightarrow \Psi[h] .
\end{aligned}
$$

In other words, it replaces the functional dependence on the space metric elements and Matter fields through the function dependence on the determinant of a space metric. Applying the transformation of variables to the $h_{i j} \rightarrow h$ Wheeler-DeWitt equation (16), one must change the functional differentiation with respect to $h_{i j}$ by the classical differentiation with respect to $h$. It can be simply done through application of the Jacobi rule for differentiation of the determinant of four-dimensional metric $\delta g=g g^{\mu v} \delta g_{\mu v}$ which leads to theresult

$$
\delta h=h h^{i j} \delta h_{i j} \rightarrow \frac{\delta}{\delta h_{i j}}=h h^{i j} \frac{\delta}{\delta h} .
$$

More detailed explanation can be found in the [1].

Making few very elementary algebraic manipulations, one obtains from the equation (16) the globally one-dimensional quantum mechanics

$$
\left(\frac{\delta^{2}}{\delta h^{2}}+V_{e f f}[h]\right) \Psi[h]=0
$$

Here $V_{\text {eff }}[h]$ is the effective potential

$$
V_{\text {eff }}[h] \equiv V_{G}[h]+V_{C}[h]+V_{M}[h],
$$

This is a simple algebraic sum of the three fundamental energetic constituents

$$
\begin{aligned}
& V_{G}[h]=-\frac{1}{6 \kappa^{2}} \frac{{ }^{(3)} R}{h}, \\
& V_{C}[h]=\frac{1}{3 \kappa^{2}} \frac{\Lambda}{h}, \\
& V_{M}[h]=\frac{1}{3 \kappa} \frac{e[h]}{h},
\end{aligned}
$$

Related to pure geometry of an embedded three-dimensional space (G), cosmological constant (C), and Matter fields (M).

On the one hand, identification of the effective potential with the square of mass of the boson $V_{\text {eff }}[h] \equiv m^{2}$ expresses the model of quantum gravity (21) as the classical theory of massive bosonic field $\Psi[h]$. The construction of quantum field theory by the method of the static Fockreper of creators and annihilators, and related thermodynamics of quantum states can be also done elementary. This part of the model of quantum gravity was discussedin the 2012' monograph by the author [1], and is not the main motive of this paper.

On the other hand, one can take into account the non-relativistic interpretation of the one-dimensional evolution (21), and treat the received global quantum gravity model as the effective onedimensional Schrödinger quantum mechanics with a certain selected effective potential being a functional of determinant of a threedimensional embedding. In the spirit of this approach the potential $V_{\text {eff }}[h]$ has intriguing meaning - (22) is the equality between "effective physics", which can be constructed by other type considerations, and three basic constituents related to an embedding space - "geometric", "cosmological", and "material".

Let us assume that the concrete form of $V_{\text {eff }}$ is fixed. Then one can express the Ricci scalar of a three-dimensional embedding as follows

$$
{ }^{(3)} R=2 \kappa e[h]+2 \Lambda-6 \kappa^{2} h V_{e f f}[h],
$$

and, therefore, the two last terms in the brackets can be treated as the dark energy density contribution

$$
e D M[h]=\frac{\Lambda}{\kappa}-3 \kappa h V_{e f f}[h] .
$$

One can list several typical examples of the physical scenarios within the global one-dimensional model of quantum gravity, with respect to the concretely fixed form of the effective gravitational potential $V_{\text {eff }}$.

1. The case of the constant non-vanishing effective gravitational potential In such a situation, the Ricci scalar curvature of the embedded space and the global one-dimensional quantum gravity aregiven by the equations

$$
\begin{aligned}
& { }^{(3)} R=2 \Lambda+2 \kappa e-6 \kappa^{2} h V_{c}, \\
& \left(\frac{\delta^{2}}{\delta h^{2}}+V_{c}\right) \Psi_{c}[h]=0,
\end{aligned}
$$

Where $\Psi_{c}[h]$ a wave is functional related to $V_{\text {eff }}=V_{c}$.

2. The case of the trivial effective gravitational potential $V_{\text {eff }}=0$. In such a situation, the three-dimensional Ricci scalar curvature and the global one-dimensional quantum gravity are

$$
{ }^{(3)} R=2 \Lambda+2 \kappa e \text {, }
$$




$$
\frac{\delta^{2}}{\delta h^{2}} \Psi_{0}[h]=0
$$

Where $\Psi_{0}$ a "free" wave is functional related to $V_{\text {eff }}=0$.

3. The case when the sum of the geometric and cosmological contributions is identically vanishing $V_{G}+V_{C}=0$, but the material contribution does not vanish identically $V_{M} \neq 0$. In such a situation, the three-dimensional Ricci scalar curvature and the global onedimensional quantum gravity are

$$
\begin{aligned}
& { }^{(3)} R=2 \Lambda, \\
& \left(\frac{\delta^{2}}{\delta h^{2}}-\frac{1}{6 \kappa^{2}} \frac{2 \kappa}{h} e[h]\right) \Psi_{M}[h]=0,
\end{aligned}
$$

Where $\Psi_{M}$ a "material" wave is functional related to the material contribution $V_{M} \neq 0$.

4. The case when the sum of the geometric and material contributions is identically vanishing $V_{G}+V_{M}=0$, while in general the cosmologicalcontribution is non-trivial $V_{C} \neq 0$. In such a situation, the Ricci scalar curvature of the embedded space and the global onedimensional quantum gravity are respectively

$$
\begin{aligned}
& { }^{(3)} R=2 \kappa e, \\
& \left(\frac{\delta^{2}}{\delta h^{2}}-\frac{1}{6 \kappa^{2}} \frac{2 \Lambda}{h}\right) \Psi_{C}[h]=0 .
\end{aligned}
$$

Here $\Psi_{C}$ is the "cosmological" wave functional related to the cosmological contribution $V_{C} \neq 0$.

5. The case when the sum of the cosmological and material contributions is identically $V_{C}+V_{M}=0$, vanishing whereas the geometric contribution is non-zero $V_{G} \neq 0$. In such a situation, the energy density of Matter fields and the global one-dimensional quantum gravity are given by the equations

$$
\begin{aligned}
& e=-\frac{\Lambda}{\kappa}, \\
& \left(\frac{\delta^{2}}{\delta h^{2}}-\frac{1}{6 \kappa^{2}} \frac{{ }^{(3)} R}{h}\right) \Psi_{G}[h]=0,
\end{aligned}
$$

Where $\Psi_{G}$ is the "geometric" wave functional related to the geometric contribution $\mathrm{V}_{\mathrm{G}} \neq 0$.

6. A more general explicit form of the effective gravitational potential can be constructed in the spirit of complex analysis. Let us consider ad hoc functional generalization of the Laurent series expansion in the global dimension $h$ of the effective gravitational potential $\mathrm{V}_{\text {eff }}[\mathrm{h}]$

$$
V_{e f f}[h]=\sum_{-\infty}^{\infty} a_{n}\left(h-h_{0}\right)^{n},
$$

in an infinitesimal neighborhood, i.e. in a one-sphere (circle) of radius $h_{\epsilon}$, of any ad hoc fixed initial value $h_{0}$ of the global dimension

$$
C\left(h_{\epsilon}\right)=\left\{h:\left|h-h_{0}\right|<h_{\epsilon}\right\} .
$$

The numbers $a_{n}$ are the series coefficients determined by the classical functional integral

$$
a_{n}=\frac{1}{2 \pi i} \int_{C\left(h_{\epsilon}\right)} \frac{V_{e f f}[h]}{\left(h-h_{0}\right)^{n+1}} \delta h,
$$

which is straightforward functional generalization of the Cauchy integral formula with the Lebesgue-Stieltjes measure-the classical functional Radon measure $\delta h$.

Let us take into considerations the most general choice of $h_{0}$ which similarly to $h$ is assumed to be a complex number. In such a situation, the Ricci scalar curvature of a three-dimensional embedded space takes the following form

$$
{ }^{(3)} R=2 \Lambda+2 \kappa e-6 \kappa^{2} \sum_{-\infty}^{\infty} b_{n}\left(h-h_{0}\right)^{n},
$$

where $b_{n}$ is the combined series coefficient

$$
b_{n}=a_{n-1}+h_{0} a_{n}=\frac{1}{2 \pi i} \int_{C\left(h_{\epsilon}\right)} \frac{h}{\left(h-h_{0}\right)^{n+1}} V_{e f f}[h] \delta h,
$$

and the global one-dimensional model of quantum gravity is then defined by the equation

$$
\left(\frac{\delta^{2}}{\delta h^{2}}+\sum_{-\infty}^{\infty} a_{n}\left(h-h_{0}\right)^{n}\right) \Psi[h]=0 .
$$

Making use of the triangle inequality one can write

$$
\left|b_{n}\right| \leq\left|a_{n-1}\right|+\left|h_{0}\right|\left|a_{n}\right|
$$

so that it can be deduced straightforwardly that

$$
\frac{\left|b_{n}\right|}{\left|a_{n}\right|} \leq \frac{\left|a_{n-1}\right|}{\left|a_{n}\right|}+\left|h_{0}\right|
$$

Applying the well-known inequality for any Riemann integral

$$
\left|\int f\right| \leq \int|f| \text {, }
$$

where $\mathrm{f}$ is considered as the Riemann-integrable function and the integral is considered as defined, to the coefficients $a_{n}$ and $b_{n}$ one obtains the inequality

$$
\left|a_{n}\right| \leq \frac{1}{h_{\epsilon}^{n+1}} \frac{1}{2 \pi} \int_{C\left(h_{\epsilon}\right)}\left|V_{e f f}\right| \delta h \leq \frac{1}{h_{\epsilon}^{n+1}}\left|a_{-1}\right|,
$$

where $a_{-1}$ is the residue of the effective gravitational potential at the fixed point $h=h_{0}$. This residue is determined by the straightforward functional generalization of the Cauchy integral formula

$$
a_{-1}=\operatorname{Res}\left(V_{e f f}, h_{0}\right)=\frac{1}{2 \pi i} \int_{C\left(h_{\epsilon}\right)} V_{e f f} \delta h,
$$

where $C\left(h_{\epsilon}\right)$ tracesoutcirclearound thefixedpoint $h_{0}$ incounterclockwise manner on the punctured disk $D=\left\{z: 0<\left|h-h_{0}\right|<R\right\}$. If the center of the circle $h-h_{0}$ is a pole of order $\mathrm{n}$, then the residue is defined by the simple limiting procedure

$$
\operatorname{Res}\left(V_{\text {eff }}, h_{0}\right)=\frac{1}{\Gamma(n)} \lim _{h \rightarrow h_{0}} \frac{\delta^{n-1}}{\delta h^{n-1}}\left(\left(h-h_{0}\right) V_{\text {eff }}\right) .
$$

Making use of the following reasoning

$$
\left|a_{n}\right|=\left|\frac{1}{2 \pi i} \int_{C\left(h_{\epsilon}\right)} \frac{V_{e f f}}{\left(h-h_{0}\right)^{n+1}}\right| \leq \frac{1}{h_{\epsilon}}\left|\frac{1}{2 \pi i} \int_{C\left(h_{\epsilon}\right)} \frac{V_{e f f}}{\left(h-h_{0}\right)^{n}}\right|=\frac{1}{h_{\epsilon}}\left|a_{n-1}\right|,
$$

one sees that for any $\mathrm{n}$ the inequality holds

$$
\frac{\left|a_{n-1}\right|}{\left|a_{n}\right|} \geq h_{\in}
$$

and hence the inequality (43) can be rewritten as 
$\frac{\left|b_{n}\right|}{\left|a_{n}\right|} \geq h_{\in}+\left|h_{0}\right|$

In the light of the triangle inequality one can write

$$
\left|b_{n+1}\right|=\left|a_{n}+h_{0} a_{n+1}\right| \leq\left|a_{n}\right|+\left|h_{0}\right|\left|a_{n+1}\right|,
$$

and because of the relation

$$
\frac{\left|a_{n+1}\right|}{\left|a_{n}\right|} \leq \frac{1}{h_{\in}}
$$

one receives the following relation

$$
\frac{\left|b_{n+1}\right|}{\left|a_{n}\right|} \leq 1+\frac{\left|h_{0}\right|}{h_{\epsilon}} \text {. }
$$

Finally, application of the inequality (50) in the equivalent form

$$
\frac{\left|a_{n}\right|}{\left|b_{n}\right|} \leq \frac{1}{h_{\epsilon}+\left|h_{0}\right|}
$$

lead us to the following upper bound

$$
\frac{\left|b_{n+1}\right|}{\left|a_{n}\right|} \frac{\left|a_{n}\right|}{\left|b_{n}\right|}=\frac{\left|b_{n+1}\right|}{\left|b_{n}\right|} \leq \frac{1}{h_{\epsilon}+\left|h_{0}\right|}\left(1+\frac{\left|h_{0}\right|}{h_{\epsilon}}\right) .
$$

Another bound for $\frac{\left|b_{n+1}\right|}{\left|b_{n}\right|}$ can be obtained as follows. In the light of the definition (43) one can write

$$
a_{n}=\frac{b_{n}-a_{n-1}}{h_{0}},
$$

and, consequently, one can deduce the recursive relation for the coefficients $b_{n}$

$$
b_{n+1}=\frac{b_{n}-a_{n-1}}{h_{0}}+h_{0} a_{n+1},
$$

which after simple algebraic manipulations leads to the relation

$$
h_{0} b_{n+1}+a_{n-1}=h_{0} a_{n+1}+b_{n} \text {. }
$$

This equation can be rewritten as

$$
1=\left|\frac{h_{0} b_{n+1}}{h_{0} a_{n+1}+b_{n}}+\frac{a_{n-1}}{h_{0} a_{n+1}+b_{n}}\right|,
$$

which after taking into account the triangle inequality gives the relation

$$
\left|\frac{h_{0} b_{n+1}}{h_{0} a_{n+1}+b_{n}}+\frac{a_{n-1}}{h_{0} a_{n+1}+b_{n}}\right| \leq\left|\frac{h_{0} b_{n+1}}{h_{0} a_{n+1}+b_{n}}\right|+\left|\frac{a_{n-1}}{h_{0} a_{n+1}+b_{n}}\right|,
$$

which leads to the conclusion

$$
\left|h_{0} a_{n+1}+b_{n}\right| \leq\left|h_{0}\right|\left|b_{n+1}\right|+\left|a_{n-1}\right| \text {. }
$$

Once again, making use of the triangle inequality one has

$$
\left|h_{0} a_{n+1}+b_{n}\right| \leq\left|h_{0}\right|\left|a_{n+1}\right|+\left|b_{n}\right|,
$$

and, consequently,

$$
\left|h_{0}\right|\left|b_{n+1}\right|-\left|b_{n}\right| \leq\left|h_{0}\right|\left|a_{n+1}\right|-\left|a_{n-1}\right| \text {. }
$$

This inequality can be rewritten in the following form

$$
\left|h_{0}\right| \frac{\left|b_{n+1}\right|}{\left|a_{n}\right|}-\frac{\left|b_{n}\right|}{\left|a_{n}\right|} \leq\left|h_{0}\right| \frac{\left|a_{n+1}\right|}{\left|a_{n}\right|}-\frac{\left|a_{n-1}\right|}{\left|a_{n}\right|},
$$

or more conveniently

$$
\left|h_{0}\right| \frac{\left|b_{n+1}\right|}{\left|b_{n}\right|}-1 \leq \frac{\left|a_{n}\right|}{\left|b_{n}\right|}\left(\left|h_{0}\right| \frac{\left|a_{n+1}\right|}{\left|a_{n}\right|}-\frac{\left|a_{n-1}\right|}{\left|a_{n}\right|}\right) .
$$

In the light of the inequality (54) and the relation

$$
\left|h_{0}\right| \frac{\left|a_{n+1}\right|}{\left|a_{n}\right|}-\frac{\left|a_{n-1}\right|}{\left|a_{n}\right|} \leq \frac{\left|h_{0}\right|}{h_{\epsilon}}-h_{\epsilon},
$$

one obtains finally the following bound

$$
\frac{\left|b_{n+1}\right|}{\left|b_{n}\right|} \leq \frac{1}{\left|h_{0}\right|}\left(1+\frac{1}{h_{\epsilon}+\left|h_{0}\right|}\left(\frac{\left|h_{0}\right|}{h_{\epsilon}}-h_{\epsilon}\right)\right),
$$

which taken together with the previous result (55) allows to deduce the inequality for the fixed point

$$
\left|h_{0}\right|\left(\left|h_{0}\right|-1\right) \geq 0 \text {, }
$$

which can be resolved immediately and gives the consistency condition for the fixed point

$$
\left|h_{0}\right| \in\{0\} \cup[1, \infty),
$$

and in itself is a non-trivial solution of the initial data problem.

Naturally, there is plenty of other possibilities for the choice of a concrete form of the effective gravitational potential $V_{\text {eff }}[h]$. However, in the next section we shall discuss only a particular situation.

\section{The invariant global dimension}

Let us note that in general the global one-dimensional quantum mechanics (21) cane be transformed by the second change of variables

$$
h \rightarrow \xi[h],
$$

where $\xi[h]$ is any functional in the global dimension $h$. In this case one can rewrite the global one-dimensional wave equation (21) in the form

$$
\left\{\left(\frac{\delta \xi[h]}{\delta h}\right)^{2} \frac{\delta^{2}}{\delta \xi[h]^{2}}+V_{\text {eff }}[\xi[h]]\right\} \Psi[\xi[h]]=0,
$$

and if the coefficient $\left(\frac{\delta \xi[h]}{\delta h}\right)^{2}$ does not vanish identically (or the transformation (70) is non-singular) then the equation (72) can be rewritten as $\xi$

$$
\left\{\frac{\delta^{2}}{\delta \xi^{2}}+V[\xi]\right\} \Psi[\xi]=0,
$$

where the new potential $V[\xi]$ is scaled effective potential $V_{\text {eff }}$ expressed by the new dimension

$$
V[\xi]=\Omega^{2}[\xi[h]] V_{e f f}[\xi[h]], \Omega[\xi[h]]=\left(\frac{\delta \xi[h]}{\delta h}\right)^{-1} .
$$

One sees easily that the following choice of the "gauge" $\xi[h]$ 


$$
\xi[h] \equiv h,
$$

Transforms the quantum mechanics (21) into itself. The choice of the transformation of variables in the form (74) is the simplest transformation of the kind $h_{i j} \rightarrow \xi\left[\operatorname{det} h_{i j}\right]$ within the WheelerDeWitt theory. Other, more advanced propositions, can be generated directly from this basic case, and should be justified by some rational arguments. Let us choose the transformation of variables in the form

$$
\xi[h]=\sqrt{h} .
$$

It is clear that this selection can be justified by the fact that $\sqrt{h}$ is the invariant volume element on an embedding with assumption that $h>00^{1}$. In this manner the dimension $\sqrt{h}$ has an invariant nature. The choice (75) yields the equation (72) with the following modified effective potential

$$
v[\xi]=4 \xi^{2} V_{e f f}[\xi] .
$$

Moreover, the singularity $\frac{1}{h}$ evidently vanishes, but actually causes that $V[\xi]$ must be studied with respect to the new "invariant" dimension $\xi$.

The very good point of reference in searching for the dimension $\xi$ is the normalization condition of the Schrödinger quantum mechanics, which for the considered situation takes the form of the LebesgueStieltjes/Radon integral

$$
\int_{\Omega\left(h_{I}, h\right)}|\Psi[\xi[h]]|^{2} \delta \xi[h]=1,
$$

Where $\Omega\left(h_{I}, h\right)$ some region of inerrability in a space of all threedimensional embedding is' s with metric $h_{i j}$ and a determinant $h=\operatorname{det} h_{i j}$. In fact this is the main condition for possible solutions of the studied model:

Proposition. Inerrability of the wave functional $\Psi[\xi]$ in the sense of functional integration in (77) determines the new dimension $\xi[h]$.

The generalized dimension $\xi[h]$ can be established in the region of inerrability $\Omega\left(h_{I}, h\right)$ as $\xi_{\Omega\left(h_{I}, h\right)}$ by using of the formula

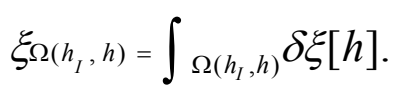

In this paper we will study few consequences of the simplest choice (74). We will use standard argument which states that the normalization condition (77) establishes inerrability constants of an arbitrary solution of the Schrödinger theory. The model in the invariant global dimension was discussed in detail in the [1].

\section{Newton-Coulomb Potential}

\section{Residual approximation}

Let us consider the situation in which the series coefficients of the effective gravitational potential are

$$
a_{n}=\left\{\begin{array}{cc}
a_{-1}=\text { const } & \text { for } n=-1 \\
0 & \text { for } n \neq-1
\end{array} .\right.
$$

We shall call such a case the residual approximation. In this approximation the effective gravitational potential (22) takes the form

$$
V_{\text {eff }}=\frac{a_{-1}}{h-h_{0}},(80)
$$

'for $h<0, \sqrt{h}$ should be replaced by $\sqrt{|h|}$, and we do not lose generality. which is formally the Newton-Coulomb potential, i.e. has the behavior like $1 / h$ where $h$ is interpreted as a kind of radial quantity. The value of the coefficient $a_{-1}$ is unknown, but it is assumed that this coefficient exists. It can be verified by straightforward easy calculation that in such a situation the combined series coefficients $b_{n}$ are

$$
b_{n}=\left\{\begin{array}{cl}
b_{-1}=h_{0} a_{-1} & \text { for } n=-1 \\
b_{0}=a_{-1} & \text { for } n=0 \\
0 & \text { for } n \neq-1,0
\end{array},\right.
$$

and, consequently, the Ricci scalar curvature of the three-dimensional space becomes

$$
{ }^{(3)} R=2 \Lambda+2 \kappa e-6 \kappa^{2} a_{-1}\left(1+\frac{h_{0}}{h-h_{0}}\right) \text {, }
$$

Where as the equation (21), defining the global one-dimensional model of quantum gravity. Takes the form

$$
\left(\frac{\delta^{2}}{\delta h^{2}}+\frac{a_{-1}}{h-h_{0}}\right) \Psi=0 .
$$

The Ricci scalar curvature (88) defines certain states of the geometry of the three-dimensional embedded space. However, even when one considers the case of vacuum, i.e. when both the energy density of Matter fields and cosmological constant are identically vanishing $e=0, \Lambda=0$, it is rather difficult to establish a three dimensional metric tensor for which the Ricci scalar curvature behaves like

$$
{ }^{(3)} R \sim 1+\frac{h_{0}}{h-h_{0}} \text {. }
$$

Interestingly, in the most general situation the residue of the threedimensional Ricci scalar curvature calculated at a fixed point $h_{0}$ is

$$
\operatorname{Res}\left({ }^{(3)} R, h_{0}\right)=2 \kappa \operatorname{Res}\left(e, h_{0}\right)-6 \kappa^{2} a_{-1} h_{0},
$$

i.e. it can be taken ad hoc identical to zero if and only if the residue of the energy density of Matter fields is

$$
\operatorname{Res}\left(e, h_{0}\right)=3 \kappa a_{-1} h_{0} \text {. }
$$

If one takes ad hoc the following relation

$$
a_{-1}=\frac{\Lambda}{3 \kappa^{2}},
$$

Then the Ricci scalar curvature of an induced three-dimensional geometry of embedded space takes the form

$$
{ }^{(3)} R=2 \kappa e-\frac{2 \Lambda h_{0}}{h-h_{0}},
$$

and its residue at a fixed point $h_{0}$

$$
\operatorname{Res}\left({ }^{(3)} R, h_{0}\right)=2 \kappa \operatorname{Res}\left(e, h_{0}\right)-2 \Lambda h_{0},
$$

Identically vanishes if and only if the residue of the energy density of Matter fields at a fixed point $h_{0}$ has the value

$$
\operatorname{Res}\left(e, h_{0}\right)=\frac{\Lambda}{\kappa} h_{0} \text {. }
$$

In such a situation, also the geometry of an embedded threedimensional space manifold is automatically Ricci-flat if and only if the energy density of Matter fields takes the following form 


$$
e=\frac{\Lambda}{\kappa} \frac{h_{0}}{h-h_{0}} .
$$

It is easy to see that another possible Ricci-flat three-dimensional manifold is obtained for the identically vanishing cosmological constant $\Lambda=0$ and the following value of the energy density of Matter fields

$$
e=3 \kappa a_{-1}\left(1+\frac{h_{0}}{h-h_{0}}\right) .
$$

\section{Newton-Coulomb quantum gravity}

In the most general situation three-dimensional spaces having induced metrics characterized by the Ricci scalar curvature of the form (84) are not yet known explicitly in literature. However, it is evident that in the particular situation $h_{0}=0$, which is fully consistent with the general condition (69), the state of affairs is determined by much more simplified equations

$$
\begin{aligned}
& { }^{(3)} R=2 \Lambda+2 \kappa e-6 \kappa^{2} a_{-1}, \\
& \left(\frac{\delta^{2}}{\delta h^{2}}+\frac{a_{-1}}{h}\right) \Psi=0 .
\end{aligned}
$$

Let us consider this particular case as the basic situation. We shall call the global one-dimensional quantum gravity described by the system of equations (93)-(94) the Newton-Coulomb quantum gravity.

As an example, we shall consider first the case of constant Ricci curvature. It is not difficult to see that in the most general view such a situationcorresponds to the identically vanishing energy density of Matter fields

$$
e \equiv 0 \text {. }
$$

We shall call such a case the Newton-Coulomb stationary quantum gravity. In such particular situation, the Ricci scalar curvature of threedimensional embedded space becomes

$$
{ }^{(3)} R=2 \Lambda-6 \kappa^{2} a_{-1}=\text { const, }
$$

and, consequently, the Ricci curvature tensor, which characterizes the intrinsic geometry of the manifolds, describes the three-dimensional Einstein manifolds [49]

$$
R_{i j}=\lambda h_{i j}
$$

where $\lambda$ the sign of the Einstein manifolds, in these specific conditions, is completely defined by parameters of the Newton-Coulomb stationary quantum gravity-the cosmological constant and the residue of the effective gravitational potential-as follows

$$
\frac{2}{3} \Lambda-2 \kappa^{2} a_{-1}=\lambda \text {. }
$$

Interestingly, the crucial consequence of identical vanishing of the energy density of Matter fields is the property of maximal symmetry of the Einstein manifolds described by the sign (98). For this reason, the Newton-Coulomb stationary quantum gravity possesses highly nontrivial geometrical interpretation: such a situation describes embedded three-dimensional manifolds which are maximally symmetric Einstein manifolds.

Consequently, one can deduce straightforwardly the classification of the three-dimensional embedded spaces, which are maximally symmetric three-dimensional Einstein manifolds (97), with respect to the value of the sign $\lambda$ (98) of a manifold in dependence on the cosmological constant and the residue $a_{-1}$ :

\section{Conclusion (Classification of maximally symmetric three-dimensional Einstein manifolds)}

The Newton-Coulomb stationary quantum gravity, defined by the effective gravitational potential $V_{\text {eff }}[h]=\frac{a_{-1}}{h}$, determines the threedimensional embedded spaces which are the maximally symmetric three-dimensional Einstein manifolds, characterized by the sign of the form (98). There is the classification of such manifolds with respect to the cosmological constant $\Lambda$ and the value $a_{-1}$ of the residue of the effective gravitational potential

1. If the sign of manifold is non-zero $\lambda \neq 0$ and the residue of the effective gravitational potential is a negative real $a_{-1}=-|\alpha|$, then the effective gravitational potential $V_{\text {eff }}[h]$ corresponds to the Newtonian attractive potential energy

$$
V_{\text {eff }}=-\frac{|\alpha|}{h} \sim-\frac{m_{1} m_{2}}{h} .
$$

a) If the cosmological constant is a positive real $\Lambda=+|\Lambda|$ then the maximally symmetric Einstein three-manifolds are characterized by the positive Ricci scalar curvature

$$
{ }^{(3)} R=\frac{2}{3}|\Lambda|+2 \kappa^{2}|\alpha| \text {. }
$$

b) If the cosmological constant is a negative real $\Lambda=-|\Lambda|$ then the maximally symmetric Einstein three-manifolds are characterized by the Ricci scalar curvature

$$
{ }^{(3)} R=-\frac{2}{3}|\Lambda|+2 \kappa^{2}|\alpha| \text {. }
$$

Which is

$$
{ }^{(3)} R \begin{cases}<0, \text { iff } & |\Lambda|>3 \kappa^{2}|\alpha| \\ =0, \text { iff } & |\Lambda|=\kappa^{2}|\alpha| \\ >0, \text { iff } & |\Lambda|<3 \kappa^{2}|\alpha|\end{cases}
$$

2. If the sign of manifold is non-zero $\lambda \neq 0$ and the residue of the effective gravitational potential is a positive real $=+||$, then the effective potential $V_{\text {eff }}[h]$ becomes the Coulomb repulsive potential energy

$$
V_{e f f}=\frac{|\alpha|}{h} \sim \frac{q_{1} q_{2}}{h} .
$$

a) If the cosmological constant is a negative real $\Lambda=-|\Lambda|$ then the maximally symmetric Einstein three-manifolds are characterized by negative Ricci scalar curvature

$$
{ }^{(3)} R=-\frac{2}{3}|\Lambda|-2 \kappa^{2}|\alpha| \text {. }
$$

b) If the cosmological constant is a positive real $\Lambda=+|\Lambda|$ then the maximally symmetric Einstein three-manifolds are characterized by the Ricci scalar curvature

$$
{ }^{(3)} R=\frac{2}{3}|\Lambda|-2 \kappa^{2}|\alpha| \text {. }
$$


Which is

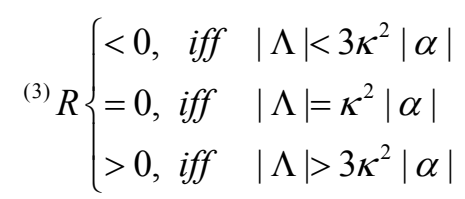

3. If the sign of manifold is identically vanishing, $\lambda=0$, i.e. the maximally symmetric Einstein three-manifolds are Ricci-flat manifolds, then one can determine uniquely the value of the residue of the effective gravitational potential as follows

$$
a_{-1}= \pm \frac{|\Lambda|}{3 \kappa^{2}} \text {. }
$$

In such a situation, one obtains the values of the cosmological constant

$$
|\Lambda| \sim\left\{\begin{array}{l}
r_{g}\left(m_{1}\right) r_{g}\left(m_{2}\right) \text { for the Newton law } \\
r_{e}\left(q_{1}\right) r_{e}\left(q_{2}\right) \text { for the Coulomb law }
\end{array}\right.
$$

where $m$ is the mass of a body generating Newtonian gravitational field in vacuum and $r_{g}(m)=\frac{2 G m}{c^{2}}$ is its gravitational radius, $q$ is the charge generating Columbic electrical field in vacuum and $r_{g}(q)=q \sqrt{\frac{G}{4 \pi \in_{0} c^{4}}}$ is its electrical radius.

Note that, in fact, by taking into account ad hoc the relation for the series coefficients (38), the residue $a_{-1}$ of the effective gravitational potential is the Cauchy integral of $V_{\text {eff }}$ at a fixed point $h_{0}=0$

$$
a_{-1}=\operatorname{Res}\left[\frac{1}{6 \kappa^{2}} \frac{1}{h}\left(-{ }^{(3)} R+2 \Lambda+2 \kappa e\right), h=0\right],
$$

and its value can be straightforwardly established as

$$
\begin{aligned}
a_{-1} & =\left.\frac{1}{6 \kappa^{2}}\left(-{ }^{(3)} R+2 \Lambda+2 \kappa e\right)\right|_{h=0} \\
& =-\frac{{ }^{(3)} R_{0}}{6 \kappa^{2}}+\frac{\Lambda}{3 \kappa^{2}}+\frac{\kappa e_{0}}{3 \kappa^{2}},
\end{aligned}
$$

where subscript " 0 " means the value of a quantity calculated in $h=0$.

Let us note that, when one shall to associate the residual effective potential $V_{\text {eff }}[h]=\frac{a_{-1}}{h}$ with any realistic quantized Kepler problem, i.e. with employing the Newtonian or the Columbic potentials, one should to identify the global dimension with a spatial distance $r=\sqrt{x^{2}+y^{2}+z^{2}}$

$$
h \equiv r \text {, }
$$

In this case, with the formal identification of the functional derivative of $\mathrm{h}$ and the classical derivative of $\mathrm{r}$, i.e. in fact the equality between the functional and classical integral measures $\delta h=d r$ wellknown in classical mechanics [50], the wave functional $\Psi[h]$ becomes the radial wave function $\Psi[r]$, and the evolution (83) becomes familiar radial type Schrödinger equation

$$
\left(\frac{d^{2}}{d r^{2}}+\frac{\mp|\alpha|}{r}\right) \Psi(r)=0
$$

Where the number $|\alpha|$ can be taken straightforwardly from the Newton law of gravitation or from the Coulomb law of electricity. The received wave equation (112) possibly describes an atomic system.

Note that there are many possible choices of the metrics $h_{i j}$ with the same value of the determinant $h=r$. For instance one can take the simple variant

$$
h_{i j}=r^{1 / 3} \delta_{i j} .
$$

However, more generally, one can parameterize the relation (111) by $\mathrm{SO}(3)$ group rotation matrix $r_{i j}: h_{i j}=r^{1 / 3} r_{i j}$, which allows use the Eulerian angles $(\theta, \varphi, \phi)$ as follows

$$
r_{i j}(\theta, \varphi, \phi) \equiv r_{i l}^{(3)}(\theta) r_{l k}^{(2)}(\varphi) r_{k j}^{(3)}(\phi),
$$

Where matrices $r_{i j}^{(p)}(\vartheta)$ are rotation matrices around the selected p-axis

$$
r_{i j}^{(3)}(\vartheta)=\left[\begin{array}{ccc}
\cos \vartheta & -\sin \vartheta & 0 \\
\sin \vartheta & \cos \vartheta & 0 \\
0 & 0 & 1
\end{array}\right], \quad r_{i j}^{(2)}(\vartheta)=\left[\begin{array}{ccc}
\cos \vartheta & 0 & \sin \vartheta \\
0 & 1 & 0 \\
-\sin \vartheta & 0 & \cos \vartheta
\end{array}\right] .
$$

This point of view was discussed in much advanced detail in the [1].

\section{Geometric Wave Functionals}

In this section we shall consider certain solutions of the global one-dimensional model of quantum gravity (21) for the case of the residual approximation of the effective gravitational potential $V_{\text {eff }}$ implemented in the previous section. In the most general situation, the considered quantum mechanical evolution

$$
\left(\frac{\delta^{2}}{\delta h^{2}} \mp \frac{|\alpha|}{h}\right) \Psi^{\mp}[h]=0,
$$

is solved by two types of wave functions $\Psi^{\mp}$ where the attractive wave functions $\Psi_{G}^{-}[h]$ are associated with the Newton-like effective gravitational potential, and the repulsive ones $\Psi^{+}[h]$ are associated with the Coulomb-like effective gravitational potential. Because of the manifest one-dimensionality of the functional evolutionary equation (116), one can solve this equation in the framework of the theory of ordinary differential equations by interpretation of the functional derivative as the ordinary one, i.e. $\frac{\delta}{\delta h}=\frac{d}{d h}$, and the wave functional as a wave function $\Psi[h]=\Psi(h)$ with no loss of generality.

In this manner, the problem to solve is given by the second order ordinary differential equation

$$
\left(\frac{d^{2}}{d h^{2}} \mp \frac{|\alpha|}{h}\right) \Psi^{\mp}[h]=0,
$$

This is well-known in the mathematical physics literature. The general solution of such a differential equation can be constructed straightforwardly by making use of the Bessel functions $J_{n}$ and $Y_{n}$ for the case of the attractive potential

$$
\Psi_{G}^{-}[h]=\sqrt{|a| h}\left[C_{1}^{-} J_{1}(2 \sqrt{|a| h})+2 i C_{2}^{-} Y_{1}(2 \sqrt{|a| h})\right],
$$

And in terms of the modified Bessel functions $I_{n}$ and $K_{n}$ for the case of repulsive potential

$$
\Psi_{G}^{+}[h]=-\sqrt{|a| h}\left[C_{1}^{+} I_{1}(2 \sqrt{|a| h})+2 C_{2}^{+} K_{1}(2 \sqrt{|a| h})\right],
$$


Where $C_{1}^{+}$and $C_{2}^{+}$are constants of integration, one takes standard definitions [51] of the Bessel functions of first and second kind, $J_{\alpha}(x)$ and $Y_{\alpha}(x)$,

$$
\begin{aligned}
& J_{\alpha}(x)=\frac{1}{\pi} \int_{0}^{\pi} d t \cos (x \cos t-\alpha t), \\
& Y_{\alpha}(x)=\frac{J_{\alpha}(x) \cos (\alpha \pi)-J_{-\alpha}(x)}{\sin (\alpha \pi)},
\end{aligned}
$$

And the modified Bessel functions of first and second kind, $I_{\alpha}(x)$ and $K_{\alpha}(x)$,

$$
\begin{aligned}
& I_{\alpha}(x)=\frac{1}{\pi} \int_{0}^{\pi} d t \exp (x \cos t) \cos (\alpha t), \\
& K_{\alpha}(x)=\frac{\pi}{2} \frac{I_{-\alpha}(x)-I_{\alpha}(x)}{\sin (\alpha \pi)} .
\end{aligned}
$$

Standardly, values of the second kind Bessel functions and modified ones for any integer's $\mathrm{n}$ can be received by application of the limiting procedure $Y_{n}(x)=\lim _{\alpha \rightarrow n} Y_{\alpha}(x), K_{n}(x)=\lim _{\alpha \rightarrow n} K_{\alpha}(x)$.

In further parts of this section we shall to present solutions of the quantum mechanics (116) with respecting of few selected boundary conditions for the general solutions (118) and (119).

\section{Boundary conditions I}

Let us consider the global one-dimensional quantum mechanics (21) with the boundary conditions for some selected initial value of the dimension $h=h_{I}$ :

$$
\Psi\left[h_{I}\right]=\Psi_{I}, \quad \frac{\delta \Psi}{\delta h}\left[h_{I}\right]=\Psi_{I}^{\prime} .
$$

With using of the regularized hyper geometric functions $p \tilde{F} q$

$$
\begin{aligned}
& p \tilde{F} q\left(\begin{array}{l}
a_{1}, \ldots, a_{p} \\
b_{1}, \ldots, b_{q}
\end{array}\right)=\frac{p \tilde{F} q\left(\begin{array}{l}
a_{1}, \ldots, a_{p} \\
b_{1}, \ldots, b_{q}
\end{array}\right)}{\Gamma\left(b_{1}\right) \ldots \Gamma\left(b_{q}\right)}, \\
& p \tilde{F} q\left(\begin{array}{l}
a_{1}, \ldots, a_{p} \\
b_{1}, \ldots, b_{q}
\end{array}\right)=\sum_{r=0}^{\infty} \frac{\left(a_{1}\right)_{r} \ldots\left(a_{p}\right)_{r} x^{r}}{\left(b_{1}\right)_{r} \ldots\left(b_{q}\right)_{r} r !}, \\
& (a)_{r} \equiv \frac{\Gamma(a+r)}{\Gamma(a)},
\end{aligned}
$$

One can write out the general solutions (118) and (119) with respect to the boundary conditions (124)

$$
\Psi_{G}^{-}=C_{1}^{-}(2 \sqrt{|\alpha| h}) K_{1}(2 \sqrt{|\alpha| h})+C_{2}^{-}(2 \sqrt{|\alpha| h})_{0}^{2} \tilde{F}_{1}\left({ }_{2}^{-} ;|\alpha| h\right),
$$

Where the sign - in the hyper geometric function notation means that all $(a)_{r}=1$, and the constants

$$
\begin{aligned}
& C_{1}^{-}=\Psi_{I 0} \tilde{F}_{1}\left(\begin{array}{l}
- \\
1
\end{array}|\alpha| h_{I}\right)-\Psi_{I}^{\prime} h_{I 0} \tilde{F}_{1}\left({ }_{2}^{-} ;|\alpha| h_{I}\right), \\
& C_{2}^{-}=\frac{1}{2}\left(\Psi_{I} K_{0}\left(2 \sqrt{|\alpha| h_{I}}\right)+\Psi_{I}^{\prime} \sqrt{\frac{h_{I}}{|\alpha|}} K_{1}\left(2 \sqrt{|\alpha| h_{I}}\right)\right),
\end{aligned}
$$

for Newtonian case, and

$$
\Psi_{G}^{+}=C_{1}^{+}(2 \sqrt{|\alpha| h}) Y_{1}(2 \sqrt{|\alpha| h})+C_{2}^{+}(2 \sqrt{|\alpha| h})_{0}^{2} \tilde{F}_{1}\left(\frac{2}{2}_{2}^{-} ;|\alpha| h\right),
$$

With constants

$$
\begin{aligned}
& C_{1}^{+}=\frac{\pi}{2}\left(\Psi_{I}^{\prime} h_{I 0} \tilde{F}_{1}\left(\frac{-}{2} ;-|\alpha| h_{I}\right)-\Psi_{I 0} \tilde{F}_{1}\left({ }_{1}^{-} ;|\alpha| h_{I}\right)\right), \\
& C_{2}^{+}=\frac{\pi}{2}\left(\Psi_{I} Y_{0}\left(2 \sqrt{|\alpha| h_{I}}\right)+\Psi_{I}^{\prime} \sqrt{\frac{h_{I}}{|\alpha|}} Y_{1}\left(2 \sqrt{|\alpha| h_{I}}\right)\right),
\end{aligned}
$$

For Columbic case.

\section{Boundary conditions II}

The second case which we want to present in this paper, are the boundary conditions for 1 st and 2 nd functional derivatives

$$
\frac{\delta \Psi}{\delta h}\left[h_{I}\right]=\Psi_{I}^{\prime}, \quad \frac{\delta^{2} \Psi}{\delta h^{2}}\left[h_{I}\right]=\Psi_{I}^{\prime \prime} .
$$

By using of the hypergeometric functions, one can express the solution for attractive case as follows

$$
\Psi_{G}^{-}=C_{1}^{-}(2 \sqrt{|\alpha| h}) K_{1}(2 \sqrt{|\alpha| h})+C_{2}^{-}(2 \sqrt{|\alpha| h})_{0}^{2} \tilde{F}_{1}\left(\frac{-}{2}_{2}^{-} ;|\alpha| h\right),
$$

Where $C_{1}^{+}$and $C_{2}^{+}$are constants defined as

$$
\begin{aligned}
& C_{1}^{-}=-h_{I}\left(\Psi_{I 0}^{\prime} \tilde{F}_{1}\left(\frac{-}{2} ;|\alpha| h_{I}\right)-\frac{\Psi_{I}^{\prime \prime}}{|\alpha|}{ }_{0} \tilde{F}_{1}\left(\begin{array}{l}
- \\
1
\end{array} ;|\alpha| h_{I}\right)\right), \\
& C_{2}^{-}=\frac{1}{2} \sqrt{\frac{h_{I}}{|\alpha|}}\left(\Psi_{I}^{\prime \prime} \sqrt{\frac{h_{I}}{|\alpha|}} K_{0}\left(2 \sqrt{|\alpha| h_{I}}\right)+\Psi_{I}^{\prime} K_{1}\left(2 \sqrt{|\alpha| h_{I}}\right)\right) .
\end{aligned}
$$

Similarly for the repulsive case one obtains easily

$$
\Psi_{G}^{+}=C_{1}^{+}(2 \sqrt{|\alpha| h}) Y_{1}(2 \sqrt{|\alpha| h})+C_{2}^{+}(2 \sqrt{|\alpha| h})_{0}^{2} \tilde{F}_{1}\left(\begin{array}{ll}
- & \\
2 & ;|\alpha| h),
\end{array}\right.
$$

With constants

$$
\begin{aligned}
& C_{1}^{+}=\frac{\pi h_{I}}{2}\left(\Psi_{I 0}^{\prime} \tilde{F}_{1}\left(\frac{-}{2} ;-|\alpha| h_{I}\right)+\frac{\Psi_{I}^{\prime \prime}}{|\alpha|}{ }_{0} \tilde{F}_{1}\left(\begin{array}{l}
- \\
1
\end{array} ;-|\alpha| h_{I}\right)\right), \\
& C_{2}^{+}=\frac{\pi}{4} \sqrt{\frac{h_{I}}{|\alpha|}}\left(\Psi_{I}^{\prime \prime} \sqrt{\frac{h_{I}}{|\alpha|}} Y_{0}\left(2 \sqrt{|\alpha| h_{I}}\right)+\Psi_{I}^{\prime} Y_{1}\left(2 \sqrt{|\alpha| h_{I}}\right)\right) .
\end{aligned}
$$

\section{Boundary conditions III}

The last possible case of boundary conditions for the considered problem is

$$
\Psi\left[h_{I}\right]=\Psi_{I}, \quad \frac{\delta^{2} \Psi}{\delta h^{2}}\left[h_{I}\right]=\Psi_{I}^{\prime \prime} .
$$

These conditions are formally improper for the problem; because of the lead to singular solutions. In this case, however, one can present the solutions in the form with formally singular constants. For the attractive potential one has

$$
\begin{aligned}
& \Psi_{G}^{-}=C_{1}^{-}(2 \sqrt{|\alpha| h}) K_{1}(2 \sqrt{|\alpha| h})+C_{2}^{-}(2 \sqrt{|\alpha| h})_{0}^{2} \tilde{F}_{1}\left(\begin{array}{l}
- \\
2
\end{array} ;|\alpha| h\right), \\
& \text { With constants }(\epsilon \rightarrow 0)
\end{aligned}
$$

$$
C_{1}^{-}=\frac{2}{\in} \sqrt{|\alpha| h_{I}}\left(\Psi_{I}-\frac{h_{I}}{|\alpha|} \Psi_{I}^{\prime \prime}\right)_{0} \tilde{F}_{1}\left(\begin{array}{l}
- \\
2
\end{array}|\alpha| h_{I}\right),
$$




$$
C_{2}^{-}=\frac{1}{\epsilon}\left(\Psi_{I}-\frac{h_{I}}{|\alpha|} \Psi_{I}^{\prime \prime}\right) K_{1}\left(2 \sqrt{|\alpha| h_{I}}\right),
$$

And similarly for the repulsive potential one obtains

$$
\Psi_{G}^{+}=C_{1}^{+}(2 \sqrt{|\alpha| h}) Y_{1}(2 \sqrt{|\alpha| h})+C_{2}^{+}(2 \sqrt{|\alpha| h})_{0}^{2} \tilde{F}_{1}\left(\begin{array}{ll}
- & \\
2 & -|\alpha| h),
\end{array}\right.
$$

With constants $(\in \rightarrow 0)$

$$
\begin{aligned}
& C_{1}^{+}=\frac{2}{\epsilon} \sqrt{|\alpha| h_{I}}\left(\Psi_{I}+\frac{h_{I}}{|\alpha|} \Psi_{I}^{\prime \prime}\right)_{0} \tilde{F}_{1}\left(\begin{array}{l}
- \\
2
\end{array}-|\alpha| h_{I}\right), \\
& C_{2}^{+}=\frac{1}{\epsilon}\left(\Psi_{I}+\frac{h_{I}}{|\alpha|} \Psi_{I}^{\prime \prime}\right) Y_{1}\left(2 \sqrt{|\alpha| h_{I}}\right),
\end{aligned}
$$
holds

However, when the following relation for the boundary conditions

$$
\pm \frac{h_{I}}{|\alpha|} \Psi_{I}^{ \pm \prime \prime}+\Psi_{I}^{ \pm} \equiv \in f \pm\left[h_{I},|\alpha|\right],
$$

Where $f \pm\left[h_{I},|\alpha|\right] \neq 0$ are some (now unknown and arbitrary) nonsingular functional of $h_{I}$ and $|\alpha|$, the sign + is related to the Newtonian case, and the sign - to the Columbic one, then the singularity of the solutions (142) and (145) is canceling. In this case the initial value $\Psi_{I}$ for the attractive case is

$$
\begin{aligned}
& \Psi_{I}^{-}=-|\alpha| h_{I 0} F_{1}\left(\begin{array}{c}
- \\
2
\end{array} ; \alpha \mid h_{I}\right)\left[C_{1}^{-}+2 \in \sqrt{|\alpha|} \int_{1}^{h_{I}} \frac{d t}{\sqrt{t}} f-[t,|\alpha|] K_{1}(2 \sqrt{|\alpha| t})\right]+ \\
& +2 \sqrt{|\alpha| h_{I}} K_{1}\left(2 \sqrt{|\alpha| h_{I}}\right)\left[C_{2}^{-}+\in|\alpha| \int_{1}^{h_{I}} d t f-[t,|\alpha|]_{0} F_{1}\left(\begin{array}{c}
- \\
2
\end{array} ;|\alpha| t\right)\right]
\end{aligned}
$$

And similarly for the repulsive one

$$
\begin{aligned}
& \Psi_{I}^{+}=|\alpha| h_{I 0} F_{1}\left(\begin{array}{l}
- \\
2
\end{array}-|\alpha| h_{I}\right)\left[C_{1}^{+}-\in \pi \sqrt{|\alpha|} \int_{1}^{h_{t}} \frac{d t}{\sqrt{t}} f+[t,|\alpha|] Y_{1}(2 \sqrt{|\alpha| t})\right]+ \\
& +2 i \sqrt{|\alpha| h_{I}} Y_{1}\left(2 \sqrt{|\alpha| h_{I}}\right)\left[C_{2}^{+}-\in \frac{i \pi}{2}|\alpha| \int_{1}^{h_{t}} d t f+[t,|\alpha|]_{0} F_{1}\left({ }_{2}^{-} ;-|\alpha| t\right)\right],
\end{aligned}
$$

Where $C_{1,2}^{ \pm}$are nonsingular constants of integration. The functional $f \pm\left[h_{I},|\alpha|\right] \neq 0$ can be established by application of the condition (148) within the general solutions (142) and (145). It yields the results

$$
\begin{aligned}
& \left.\Psi_{I}^{-}=8|\alpha| h_{I} K_{1}\left(2 \sqrt{|\alpha| h_{I}}\right)_{0} \tilde{F}_{1}\left(\begin{array}{l}
- \\
2
\end{array}\right) f \mid h_{I}\right) f-\left[h_{I},|\alpha|\right], \\
& \Psi_{I}^{+}=8|\alpha| h_{I} Y_{1}\left(2 \sqrt{|\alpha| h_{I}}\right)_{0} \tilde{F}_{1}\left(\begin{array}{c}
- \\
2
\end{array}-|\alpha| h_{I}\right) f+\left[h_{I},|\alpha|\right] .
\end{aligned}
$$

Employing straightforwardly these results into the equalities (149) and (150) one obtains the integral equations for the functional $f \pm$. For the columbic situation one receives the following equation

$$
\begin{aligned}
& -\frac{C_{1}^{-}}{4}\left(2 \sqrt{|\alpha| h_{I}}\right)_{0} F_{1}\left({ }_{2}^{-} ;|\alpha| h_{I}\right)+C_{2}^{-} K_{1}\left(2 \sqrt{|\alpha| h_{I}}\right)+
\end{aligned}
$$

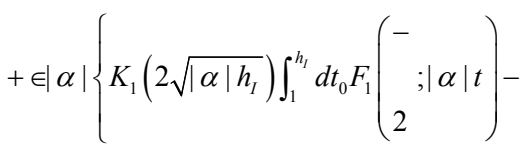

$$
\begin{aligned}
& \left.-\sqrt{h_{I}} F_{1}\left(\begin{array}{l}
- \\
2
\end{array}|\alpha| h_{I}\right) \int_{1}^{h_{I}} \frac{d t}{\sqrt{t}} K_{1}(2 \sqrt{|\alpha| t})\right\} f-[t,|\alpha|]= \\
& =2\left(2 \sqrt{|\alpha| h_{I}}\right) K_{1}\left(2 \sqrt{|\alpha| h_{I}}\right)_{0} \tilde{F}_{1}\left(\begin{array}{l}
- \\
2
\end{array}|\alpha| h_{I}\right) f-\left[h_{I},|\alpha|\right] \text {, }
\end{aligned}
$$

And similarly for the Newtonian case one derives the relation

$$
\begin{aligned}
& \frac{C_{1}^{+}}{4}\left(2 \sqrt{|\alpha| h_{I}}\right)_{0} F_{1}\left(\begin{array}{c}
- \\
2
\end{array}-|\alpha| h_{I}\right)+i C_{2}^{+} Y_{1}\left(2 \sqrt{|\alpha| h_{I}}\right)- \\
& -\in \frac{\pi}{2}|\alpha|\left\{\sqrt{h_{I}} F_{1}\left(\begin{array}{c}
- \\
2 \\
2
\end{array}-|\alpha| h_{I}\right) \int_{1}^{h_{I}} \frac{d t}{\sqrt{t}} Y_{1}(2 \sqrt{|\alpha| t})+\right. \\
& \left.+i Y_{1}\left(2 \sqrt{|\alpha| h_{I}}\right) \int_{1}^{h_{I}} d t_{0} F_{1}\left(\begin{array}{c}
- \\
2
\end{array}-|\alpha| t\right)\right\} f+[t,|\alpha|]= \\
& \left.=2\left(2 \sqrt{|\alpha| h_{I}}\right) Y_{1}\left(2 \sqrt{|\alpha| h_{I}}\right)_{0} \tilde{F}_{1}\left(\begin{array}{l}
- \\
2
\end{array}\right)-|\alpha| h_{I}\right) f+\left[h_{I},|\alpha|\right] .
\end{aligned}
$$

In both the cases the integral operators acting on the functional $f \pm$ are nonsingular. By this reason one can put straightforwardly the formal limit $(\in \rightarrow 0)$ in the integral equations (153) and (154), and by doing few elementary algebraic manipulations one can extract the searched functionals. The final results are as follows

$$
\begin{aligned}
& f_{-}\left[h_{I},|\alpha|\right]=\frac{-C_{1}^{-} / 8}{K_{1}\left(2 \sqrt{|\alpha| h_{I}}\right)}+\frac{C_{2}^{-} / 4}{I_{1}\left(2 \sqrt{|\alpha| h_{I}}\right)}, \\
& f_{+}\left[h_{I},|\alpha|\right]=\frac{C_{1}^{+} / 8}{Y_{1}\left(2 \sqrt{|\alpha| h_{I}}\right)}+\frac{i C_{2}^{+} / 4}{J_{1}\left(2 \sqrt{|\alpha| h_{I}}\right)},
\end{aligned}
$$

In this manner the initial data conditions for the considered boundary conditions (141) cannot be chosen arbitrary, but according to the rules

$$
\begin{aligned}
& \Psi_{I}^{-}=\sqrt{|\alpha| h_{I}}\left[-C_{1}^{-} I_{1}\left(2 \sqrt{|\alpha| h_{I}}\right)+2 C_{2}^{-} K_{1}\left(2 \sqrt{|\alpha| h_{I}}\right)\right], \\
& \Psi_{I}^{+}=\sqrt{|\alpha| h_{I}}\left[C_{1}^{+} J_{1}\left(2 \sqrt{|\alpha| h_{I}}\right)+2 i C_{2}^{+} Y_{1}\left(2 \sqrt{|\alpha| h_{I}}\right)\right] .
\end{aligned}
$$

The supposed equation for boundary values (148) is not unique, and can be replaced by other conditions. The discussed case, however, reflects the typical questions arising within the problem.

\section{Discussion}

In this paper we have discussed the quantum mechanical model of quantum gravity arising from the global one-dimensional conjecture within quantum general relativity considered recently by the author [1]. This model straightforwardly bases on the effective potential (22) being 
Citation: Glinka LA (2014) On The Residual Effective Potential within Global One-Dimensional Quantum Gravity. J Astrophys Aerospace Technol 2: 103. doi:10.4172/2329-6542.1000103

Page 11 of 11

a simple algebraic sum of three fundamental energetic constituents "geometric", "cosmological", and "material", with nontrivial change in potential behavior with respect to the initial model that was the Wheeler-DeWitt quantum Geometrodynamics (16). The relation between the models is established by change of both the differential operator and the potential.

We have considered the analytical form of the effective potential, and concentrated an especial attention on the physical conclusions following from the residual effective potential, which on some wellestablished conventional level is directly identified with the attractive Newton's gravitation or the repulsive Coulomb's electrostatics. Studying of this special case allowed concluding that in the case of Matter fields's energy absence, in the global one-dimensional model of quantum gravity, the maximally symmetric three-dimensional Einstein manifolds are the characteristic embedding for the residual effective potential. Finally, we have found some solutions of the model of quantum gravity in the residual approximation.

We hope to discuss further conclusions of the global-one dimensional model of quantum gravity in the next contributions.

\section{References}

1. Glinka LA (2012) Aethereal Multiverse: A New Unifying Theoretical Approach to Cosmology, Particle Physics, and Quantum Gravity.

2. Glinka (2010) Global One-Dimensionality Conjecture within Quantum General Relativity. 16: 7-15.

3. Avramidi IG (2000) Heat Kernel and Quantum Gravity.

4. Callender C, Huggett N (2001) Physics meets philosophy at the Planck scale. Contemporary theories in quantum gravity.

5. Kursunoglu BN, Mintz SL, Perlmutter A (2002) Quantum Gravity, Generalized Theory of Gravitation and Superstring Theory Based Unification.

6. Louko J (2005) Quantum Gravity. From Theory To Experimental Search.

7. RovelliC (2004) Quantum Gravity.

8. GomberoffA, Marolf D (2005) Lectures on Quantum Gravity.

9. Rickles D, French S, Saatsi JT (2006) The Structural Foundations of Quantum Gravity.

10. Kiefer C (2007) Quantum Gravity.

11. Thiemann T (2007) Modern Canonical Quantum General Relativity.

12. Oriti D (2009) Approaches to Quantum Gravity. Toward a New Understanding of Space, Time, and Matter.

13. Einstein A( 1914) Phys. Z. 15, 176; Sitzungsber. Preuss. Akad. Wiss.Berlin 2, 1030 (1914); ibid. 44, 778 (1915); ibid. 46, 799 (1915); ibid.48, 844 (1915); ibid. 2, 1111 (1916); ibid. 1, 142 (1917); ibid. 1, 349(1919); Ann. Phys. 49, 769 (1916); ibid. 55, 241 (1918); Math. Ann. 7,99 (1926).

14. Hilbert D (1915) Nachr. Ges. Wiss. Göttingen 27, 395; ibid. 61, 53 (1917).

15. Riemann B (1920) Nachr. Ges. Wiss. Göttingen 13, 133.

16. Palatini A (1919) Deduzioneinvariantivadelleequazionigravitazionali dal principio di Hamilton. Rend. Circ. Mat. Palermo 43: 203-212.

17. York JW (1972) Phys. Rev. Lett. 28, 1082.

18. Gibbons GW, Hawking SW (1977) Action integrals and partition functions in quantum gravity. Phys. Rev. D 15: 2752

19. Nash JF (1952) Ann. Math. 56, 405; ibid. 63, 20 (1956)

20. Kowalczyk A (1981) Bull. Acad. Polon. Sci. Ser. Sci. Math. 28, 385.

21. Masahiro S (1987) Nash Manifolds.1269: 1-215.

22. Günther M (1989) Zum Einbettungssatz von J. Nash. Math. Nachr 144: 165-187.

23. Arnowitt R, Deser S, Misner CW, Bryce S (1962) Gravitation: An Introduction to Current Research.
24. DeWitt BS (2003) The Global Approach to Quantum Field Theory.

25. Poisson E (2004) A Relativist's Toolkit. The Mathematics of Black-Hole Mechanics.

26. Hanson A, ReggeT, Teitelboim C (1976) Constrained Hamiltonian Systems.

27. DeWitt BS (1967) Quantum Theory of Gravity. I. The Canonical Theory.

28. Dirac PAM Lectures on Quantum Mechanics (Belfer Graduate School of Science, Yeshiva University, 1964); Phys. Rev. 114, 924 (1959); Phys. Rev. Lett. 2, 368 (1959); Proc. Roy. Soc. (London) A 246, 326 (1958); Can. J. Math 2, 129 (1950)

29. Fischer AE,Carmeli M, Fickler SI (1969) Relativity. Proceedings of the Relativity Conference in the Midwest held at Cincinnati, Ohio, June 2-6, 1969, pp. 303359 (Plenum Press, 1970); Gen. Rel. Grav. 15, 1191 (1983), J. Math. Phys. 27, 718 (1986).

30. Carmeli M, DeWitt BS, Fickler SI (1969) Relativity. Proceedings of the Relativity Conference in the Midwest held at Cincinnati, Ohio, June 2-6, 1969, pp. 359 374 (Plenum Press, 1970)

31. Giulini D (2009) The Superspace of Geometrodynamics. Gen RelGrav41: 785 815

32. Faddeev LD (1982) The energy problem in Einstein's theory of gravitation. Usp Fiz Nauk 25: 130-142.

33. Wheeler JA (1957) On the nature of quantum Geometrodynamics. Ann Phys 2: 604-614.

34. Hall MJW (2005) Exact uncertainty approach in quantum mechanics and quantum gravity. Gen Rel Grav 37: 1505-1515.

35. Henriques AB (2006) Loop quantum cosmology and the Wheeler-De Witt equation. Gen Rel Grav 38: 1645-1659.

36. Kamenshchik AY, Kiefer C, Sandhöfer B(2007) Quantum cosmology with a big-brake singularity. Phys Rev D

37. Carroll R (2007) Metric fluctuations, entropy, and the Wheeler-deWitt equation Theor Math Phys 152: 904-914.

38. Barbero JFG (2008) Quantum Geometry and Quantum Gravity. AIP Conf Proc. 39. Thiemann T (2008) Loop Quantum Gravity. Int J Mod Phys A

40. DamourT, Nicolai H (2008) Symmetries, Singularities And The De-Emergence Of Space. Int J Mod Phys D.

41. Hartle JB, Hawking SW, Hertog T (2008) Classical universes of the noboundary quantum state. Phys Rev Lett.

42. Kiefer C (2009) Quantum geometrodynamics: whence, whither? Gen Rel Grav 41: 877-901.

43. Hawking SW (1979) General Relativity: An Einstein centenary survey. Commun. Math. Phys. 43, 199 (1975); ibid. 55, 133 (1977); Phys. Rev. D 13 191 (1976); ibid. 14, 2460 (1976); ibid. 18, 1747 (1978); ibid. 32, 259 (1985) ibid. 37, 904 (1988); Phys. Lett. B 134, 403 (1984); Nucl. Phys. B 239, 257 (1984); Phys. Scr. T 117, 49 (2005); contributions in: Hawking SW, Israel W, pp. 746-785 (Cambridge University Press, 1979);

44. Levy ME, Deser S (1979) Recent Developments in Gravitation: Cargese 1978 45. DeWitt BS, StoraR (1984) Relativity, Groups and Topology II.

46. de Vega HJ, Sánchez N (1986) Field Theory, Quantum Gravity, and Strings Proceedings of a Seminar Series Held at DAPHE, Observatoire de Meudon, and LPTHE, Université Pierre et Marie Curie, Paris, Between October 1984 and October 1985

47. Halliwell JJ, Perez-Marcader J, Zurek WH (1992) Physical Origins of Time Assymetry.

48. Hawking SW, Israel W (1987) Three hundred years of gravitation.

49. Besse AL (1987) Einstein Manifolds.

50. Goldstein H, Poole CP, Safko JL (2000) Classical Mechanics.

51. Andrews GE, Askey R, Roy R (2000) Special Functions. 